\title{
Covid-19 related hospital admissions in the United States: needs and outcomes
}

\author{
Lessons from rigorous inpatient studies will inform long term follow-up and continuing care
}

\author{
George L Anesi instructor of medicine ${ }^{1}$, Scott D Halpern professor of medicine ${ }^{2}, \mathrm{M}$ Kit Delgado \\ assistant professor of emergency medicine ${ }^{3}$
}

\begin{abstract}
${ }^{1}$ Palliative and Advanced Illness Research (PAIR) Center, Division of Pulmonary, Allergy, and Critical Care, University of Pennsylvania Perelman School of Medicine, Philadelphia, PA, USA; ${ }^{2}$ Palliative and Advanced Illness Research (PAIR) Center, University of Pennsylvania Perelman School of Medicine, Philadelphia, PA, USA; ${ }^{3}$ Center for Emergency Care Policy and Research, Department of Emergency Medicine, University of Pennsylvania Perelman School of Medicine, Philadelphia, PA, USA
\end{abstract}

Cohort studies and case series of patients with coronavirus disease 2019 (covid-19) have spread almost as quickly as the virus, ${ }^{1-5}$ with inadequate methodological quality sometimes hampering thoughtful interpretation. For example, one New York cohort study initially reported a mortality of $88 \%$ for patients requiring mechanical ventilation before readers' responses led to a correction clarifying that the rate was $25 \%$ and would grow to an uncertain extent because $72 \%$ of patients were still in hospital. ${ }^{4}$ In contrast, the two linked cohort studies from the United States provide rigorous outcome ascertainment and adjustment for case mix. ${ }^{67}$ By delaying publication until most enrolled patients had confirmed outcomes and by using modern survival analysis methods these authors have greatly enhanced our understanding of the epidemiology of covid-19. Lewnard and colleagues (doi:10.1136/bmj.m1923) report on 1840 people with covid-19 at Kaiser Permanente system hospitals in California and Washington state. ${ }^{6}$ Median age was 61 years with $25 \%$ being 48 years or younger. The authors used survival analysis methods adjusted for patient characteristics to account for those who remained in hospital at the time of analysis. The study reported incidence of hospital admission (15-23 episodes per 100000 cohort members in their insured populations), hospital length of stay (median 10.1 days), probability of intensive care unit (ICU) admission (41\%), length of stay among ICU patients (median 10.5 days, and mortality. Mortality among all patients with completed hospital stays and outcomes was $18 \%$, with risk factors including increased age and male sex.

The study also estimated dynamic changes in infection transmission rates by calculating the effective reproduction number $\left(R_{E}\right)$. An $R_{E}>$ greater than 1 indicates that an individual infects more than one other individual in a given population on a given day. The authors estimated a high $R_{E}$ ranging from 1.31 to 2.49 for those acquiring infection on 1 March 2020 in the different regions covered by the study and lower $R_{E}$ ranging from 0.78 to 0.90 for those acquiring infection on 1 April 2020. This strong evidence of decreased transmission over time corresponds with enactment of social distancing policies.

Petrilli and colleagues (doi:10.1136/bmj.m1966) report on 5279 patients with covid-19 at an academic medical center in New York City. ${ }^{7}$ The cohort included patients testing positive for severe acute respiratory syndrome coronavirus 2 (SARS-CoV-2), the virus responsible for covid-19, in any outpatient or emergency department setting. Median age of patients admitted to hospital was (62 years with $25 \%$ being 51 years or younger). The study outcomes included inpatient hospital stay (52\%) and critical illness among those admitted to hospital (36\%), representing a composite of ICU admission, mechanical ventilation, and hospital mortality or discharge to hospice care. Mortality among patients receiving mechanical ventilation was $60 \%$. Of the 990 patients who developed critical illness, 399 $(40 \%)$ were younger than 65 years with $91(9 \%)$ patients being younger than 45 years. Of the entire cohort, outcome assessment was complete for $99.6 \%$ of patients, greatly enhancing confidence in these estimates.

Several patient characteristics in the New York study, including age, male sex, obesity, and heart failure were risks for more severe illness, mirroring earlier reports. ${ }^{145}$ The authors also provide compelling evidence confirming the predictive value of reduced oxygen saturation and increases in troponin, $\mathrm{C}$ reactive protein, and D-dimer levels near admission. As in previous reports, asthma and chronic obstructive pulmonary disease were notably absent from identified risk factors. Importantly, although emerging data show rates of covid-19 cases, hospital admissions, and deaths among African-American and Latino populations have been worse than among white populations, ${ }^{89}$ the authors did not find evidence of racial or ethnic disparities in critical illness or death once patients had been admitted to hospitals in their health system. 
Perhaps the most intriguing finding from the New York cohort was that risk of critical illness declined progressively over the study period, with a suggestion of declining mortality as well, without changes in risk of hospital admission. Several potential explanations worthy of future investigation include the influence of strain in hospital capacity on quality of care, allocation of resources, and disposition decisions in the emergency department ${ }^{1011}$; changes in care delivery over time, such as proning in awake, non-intubated patients to avoid intubation ${ }^{12}$ or better adherence to lung protective mechanical ventilation strategies; and changes in targeted therapy that might be beneficial (remdesivir ${ }^{13}$ and anticoagulation ${ }^{14}$ ) or harmful (hydroxychloroquine $^{15}$ ).

These studies provide the strongest evidence to date that for every covid-19 related hospital death in the US there have been four times as many survivors, typically requiring one to two weeks in hospital, and often in the ICU. The studies also provide rigorous confirmation of risk factors for poor outcomes that had been suggested in early reports. While older age is the greatest risk factor for adverse outcomes, including death, the burden of severe covid-19 illness is clearly borne across the lifespan, as most hospital admissions $\left(53 \%^{7}\right.$ to $\left.60 \%{ }^{6}\right)$ occurred in patients younger than 65 years. Early reports with incomplete follow-up are liable to considerable errors in reporting absolute event rates but often provide reasonable estimates of relative risks. These lessons will inform the transition to handling long term debility among hundreds of thousands of hospital survivors, deciding which patients can be managed safely at home, understanding risks for readmission, and developing effective strategies for long term care.

Competing interests: The BMJ has judged that there are no disqualifying financial ties to commercial companies. The authors declare the following other interests: GLA received a grant from the Agency for Healthcare Research and Quality (K12HS026372) and reports pending fees from UpToDate for authoring covid-19 related educational content. SDH reports no other interests. MKD received a grant from the National Institutes of Health (K23HD090272001) and reports receiving a travel honorarium for participating in an expert panel on innovative approaches to acute pain management for United Health Group. The views are those of the authors and do not represent those of the funders. The BMJ policy on financial interests is here: https://www.bmj.com/sites/default/files/attachments/resources/ 2016/03/16-current-bmj-education-coi-form.pdf.

Provenance and peer review: Commissioned; not peer reviewed.

Wang D, Hu B, Hu C, etal . Clinical characteristics of 138 hospitalized patients with 2019 novel coronavirus-infected pneumonia in Wuhan, China. JAMA 2020;323:1061-9. 10.1001/jama.2020.1585 32031570

2 Grasselli G, Zangrillo A, Zanella A, etal. COVID-19 Lombardy ICU Network. Baseline characteristics and outcomes of 1591 patients infected with SARS-CoV-2 admitted to ICUs of the Lombardy region, Italy. JAMA 2020;323:1574-81.

10.1001/jama.2020.5394 32250385

3 Bhatraju PK, Ghassemieh BJ, Nichols M, etal . Covid-19 in critically ill patients in the Seattle region-case series. N Engl J Med 2020;382:2012-22. 10.1056/NEJMoa2004500 32227758

4 Richardson S, Hirsch JS, Narasimhan M, etal. and the Northwell COVID-19 Research Consortium. Presenting characteristics, comorbidities, and outcomes among 5700 patients hospitalized with COVID-19 in the New York City area. JAMA 2020, published online 22 April. 10.1001/jama.2020.6775 32320003

5 Cummings MJ, Baldwin MR, Abrams D, etal . Epidemiology, clinical course, and outcomes of critically ill adults with COVID-19 in New York City: a prospective cohort study. Lancet 2020, published online 19 May. 10.1016/S0140-6736(20)31189-2 32442528

6 Lewnard JA, Liu VX, Jackson ML, etal . Incidence, clinical outcomes, and transmission dynamics of severe coronavirus disease 2019 in California and Washington: prospective cohort study. BMJ 2020;369:m1923. 10.1136/bmj.m1923 32444358

7 Petrilli CM, Jones SA, Yang J, etal . Factors associated with hospital admission and critical illness among 5279 people with coronavirus disease 2019 in New York City: prospective cohort study. BMJ 2020;369:m1966. 10.1136/bmj.m1966 32444366

8 Azar KMJ, Shen Z, Romanelli RJ, etal . Disparities In Outcomes Among COVID-19 Patients In A Large Health Care System In California. Health Aff (Millwood) 2020; $\{202000598.32437224$

9 Webb Hooper M, Nápoles AM, Pérez-Stable EJ. COVID-19 and Racial/Ethnic Disparities. JAMA 2020. 32391864

10 Anesi GL, Liu VX, Gabler NB, etal . Associations of Intensive Care Unit Capacity Strain with Disposition and Outcomes of Patients with Sepsis Presenting to the Emergency Department. Ann Am Thorac Soc 2018;15:1328-35. 10.1513/AnnalsATS.201804-241OC 30113865

11 Weissman GE, Gabler NB, Brown SE, Halpern SD. Intensive care unit capacity strain and adherence to prophylaxis guidelines. J Crit Care 2015;30:1303-9. 10.1016/j.jcrc.2015.08.015 26376062

12 Caputo ND, Strayer RJ, Levitan R. Early Self-Proning in Awake, Non-intubated Patients in the Emergency Department: A Single ED's Experience During the COVID-19 Pandemic. Acad Emerg Med 2020;27:375-8. 10.1111/acem.13994 32320506

13 Beigel JH, Tomashek KM, Dodd LE, et al, for the ACTT-1 Study Group Members. Remdesivir for the treatment of covid-19 - preliminary report. NEJM $2020 \mathrm{https}: / / \mathrm{www}$. nejm.org/doi/full/10.1056/NEJMoa2007764

14 Kollias A, Kyriakoulis KG, Dimakakos E, Poulakou G, Stergiou GS, Syrigos K. Thromboembolic risk and anticoagulant therapy in COVID-19 patients: emerging evidence and call for action. Br J Haematol 2020, published online 18 April. 10.1111/bjh.16727 32304577

15 Mehra MR, Desai SS, Ruschitzka F, Patel AN. Hydroxychloroquine or chloroquine with or without a macrolide for treatment of COVID-19: a multinational registry analysis. Lancet 2020, published online 22 May. 10.1016/S0140-6736(20)31180-6. 32450107

Published by the BMJ Publishing Group Limited. For permission to use (where not already granted under a licence) please go to http://group.bmj.com/group/rights-licensing/ permissions 